\title{
Respiratory and pulmonary complications in head and neck cancer patients: An evidence-based review for the COVID-19 era
}

\author{
Stephen Y. Kang, MD ${ }^{1}$ \\ ${ }^{1}$ The James Cancer Hospital and Solove Research Institute
}

April 28, 2020

\begin{abstract}
Background: Pulmonary complications and infections frequently affect patients with head and neck squamous cell carcinoma (HNSCC). Common characteristics can predispose these patients to the development of severe respiratory illness, which may be particularly relevant during the 2019 coronavirus disease (COVID-19) pandemic. Methods: A scoping review was performed to assess the impact of pulmonary comorbidities and adverse respiratory outcomes in HNSCC patients. Results: Advanced age, history of tobacco and alcohol abuse, and cardiopulmonary comorbidities are significant risk factors for the development of adverse respiratory outcomes. Treatment toxicities from radiation or chemoradiation therapy significantly increase these risks. Conclusion: Respiratory complications are a frequent cause of morbidity and mortality among HNSCC patients, and the COVID-19 pandemic may disproportionately affect this population. Interventions designed to decrease smoking and alcohol use, improve oral hygiene, and aggressively manage medical comorbidities are important to the long-term management and health of these patients.
\end{abstract}

Dustin A. Silverman, $\mathrm{MD}^{1 *}$; Chen Lin, MD ${ }^{1 *}$; Akina Tamaki, $\mathrm{MD}^{1}$; Sidharth V. Puram, MD, PhD ${ }^{2}$; Ricardo L. Carrau, MD ${ }^{1}$; Nolan B. Seim, $\mathrm{MD}^{1}$; Antoine Eskander, MD, ScM ${ }^{3}$; James W. Rocco, MD, $\mathrm{PhD}^{1}$; Matthew O. Old, MD ${ }^{1}$; Stephen Y. Kang, $\mathrm{MD}^{1}$

Author Affiliations: 1 Department of Otolaryngology-Head \& Neck Surgery, Division of Head \& Neck Oncology, The Ohio State University, James Cancer Center and Solove Research Institute, Columbus, OH, USA 2 Department of Otolaryngology-Head \& Neck Surgery and Department of Genetics, Washington University School of Medicine, Ear, Nose \& Throat Center, 4921 Parkway Place, 11th Floor, St. 14 Louis, MO 63110

Department of Otolaryngology-Head and Neck Surgery, Sunnybrook Health Sciences Center, 162075 Bayview Avenue, Suite M1-102, Toronto, ON, M4N 3M5

*These authors contributed equally to this manuscript

Word Count: 3188

Funding: None

Conflicts of Interest: Antoine Eskander MD, ScM is a paid consultant for Bristol-Myers and has received research support from Merck. "None declared" for all remaining authors.

\section{Corresponding Author:}

Stephen Y. Kang, MD

Department of Otolaryngology-Head \& Neck Surgery 
The James Cancer Hospital and Solove Research Institute

$460 \mathrm{~W}$ 10th Avenue, 5th Floor

Columbus, OH 43210

Telephone: 614-685-6778 40 Fax: 614-293-7292 (Attn: Elaine Ortiz)

Stephen.kang@osumc.edu

\section{ABSTRACT:}

Background: Pulmonary complications and infections frequently affect patients with head and neck squamous cell carcinoma (HNSCC). Common characteristics can predispose these patients to the development of severe respiratory illness, which may be particularly relevant during the 2019 coronavirus disease (COVID19) pandemic.

Methods: A scoping review was performed to assess the impact of pulmonary comorbidities and adverse respiratory outcomes in HNSCC patients.

Results: Advanced age, history of tobacco and alcohol abuse, and cardiopulmonary comorbidities are significant risk factors for the development of adverse respiratory outcomes. Treatment toxicities from radiation or chemoradiation therapy significantly increase these risks.

Conclusion: Respiratory complications are a frequent cause of morbidity and mortality among HNSCC patients, and the COVID-19 pandemic may disproportionately affect this population. Interventions designed to decrease smoking and alcohol use, improve oral hygiene, and aggressively manage medical comorbidities are important to the long-term management and health of these patients.

\section{INTRODUCTION:}

The novel severe acute respiratory syndrome coronavirus 2 (SARS-CoV-2) and associated 2019 coronavirus disease (COVID-19) pandemic has created unique challenges for all healthcare providers and patients. Complications resulting from COVID-19 infection are significant, and immunocompromised patients, including cancer patients, appear particularly susceptible. Limited data regarding the effect on immunocompromised patients is available. Early reviews indicate that severe respiratory events including pneumonia, need for mechanical ventilation, intensive care unit (ICU) admission, and increased risk of death may be experienced by a significant proportion of patients with cancer. ${ }^{1,2}$

Patients with head and neck squamous cell carcinoma (HNSCC) share common characteristics, namely upper aerodigestive tumors, advanced age, history of tobacco and alcohol abuse, and multiple cardiopulmonary comorbidities which predispose to severe adverse respiratory outcomes. Moreover, potential toxicities conferred by chemotherapy and radiation therapy (RT) have been demonstrated to significantly increase rates of dysphagia, aspiration, and pneumonia within this population. ${ }^{3-6}$ Recent studies have sought to identify sources of mortality and characterize long-term morbidity following HNSCC treatment. ${ }^{6-8}$ An understanding of the various adverse respiratory outcomes experienced among head and neck patients following treatment, particularly during the COVID-19 pandemic, is critical in treatment algorithms and medical decision-making.

A scoping review ${ }^{9}$ was performed to assess the impact of pneumonia, aspiration pneumonitis, and other select viral and bacterial infectious etiologies in patients with HNSCC. An appraisal of risk factors, sources of competing mortality, pathophysiology, and respiratory outcomes is also provided. 


\section{COVID-19 and Cancer}

Cancer patients and immunocompromised hosts are particularly susceptible to lung infection. The spectrum of various infectious etiologies ranges widely from viral, bacterial, and fungal pathogens; however, patients' unique treatment regimens and resulting immunologic deficits predispose patients to particular infections. ${ }^{10-14}$ In the setting of SARS-CoV-2 and the COVID-19 pandemic, increased emphasis is being placed upon the treatment and protection of those with cancer who may be at increased risk to develop severe and potentially life-threatening respiratory infections. ${ }^{15}$

Following the COVID-19 outbreak, the Italian National Institute of Health established a surveillance program to collect information on all patients with reverse transcriptase-polymerase chain reaction (RT-PCR) confirmed SARS-CoV-2 infection. Among the Italian population, the overall case fatality rate (CFR) was $7.2 \%$ (1625 deaths/22,512 cases) as of March $15,2020 .{ }^{16}$ In a subsample of 355 patients, $20.3 \%$ were noted to have active cancer in addition to multiple comorbidities. ${ }^{17}$ Although a higher mean age (79.5 \pm 8.1 years) was observed within this cohort, separate analyses have reported a fatality rate approximating $20 \%$ in patients aged 80 and older. ${ }^{18}$ While CFR estimates and degrees of morbidity may vary based upon testing strategies and population demographics, cancer patients and elderly individuals appear at higher risk for adverse outcomes from COVID-19 infection.

Early reports from China indicate that infected cancer patients have a 3.5 times higher risk of requiring ICU admission, mechanical ventilation, or death compared to individuals without cancer. ${ }^{1}$ Zhang et al. noted a strong association between anti-cancer therapy and risk of severe effects from COVID-19 infection (HR $4.079, p=0.037)$ with 6 of $28(21.4 \%)$ COVID-19 patients having received immunosuppressive treatment within the last 14 days. $^{2}$ In a separate prospective analysis of 1,590 patients with laboratory-confirmed COVID-19 disease, Liang et al. discovered that 18 (1\%) patients had a history of cancer, a rate which appears to be higher than the overall cancer incidence within the Chinese population $(0.29 \%)$ according to 2015 epidemiologic data. ${ }^{1}$ Of the 16 patients with a known oncologic treatment, $25 \%$ underwent surgery or received chemotherapy within the past month. Compared to patients without cancer in this study, oncology patients were older (mean 63.1 years vs. 48.7 years) and more likely to have a history of smoking (22\%). Notably, these characteristics are also shared among a large proportion of patients with HNSCC. In a multivariable model, cancer history was associated with the highest risk for severe events (OR 5.4, $p<$ 0.01). Older age, while not significant, was the only risk factor associated with increased risk for severe events in the cancer group (OR 1.43). Critics of this analysis argue that exposure to an infectious source, rather than an association with cancer, is the most important comorbid factor given that 12 of the 18 patients had recovered from their initial cancer treatments (e.g. surgery or chemotherapy) and were without obvious immunosuppression. ${ }^{19}$ The response, by Wang and Zhang, further notes that a higher median patient age of those with cancer (63.1 vs. 48.7) in addition to increased comorbidities, remain significant factors associated with worse COVID-19 outcomes. These findings have been supported by prior COVID-19 analyses. ${ }^{20-22}$

Although data regarding the impact of prior viral outbreaks on patients with HNSCC is lacking, the decision to proceed with anti-cancer therapy, including surgery, in the current context of COVID-19 should be carefully considered given the risk of severe respiratory complications, immunosuppression, and death within this cohort. A recent review of 34 asymptomatic patients who underwent various elective surgeries during the COVID-19 incubation period exemplifies these risks. ${ }^{23}$ All 34 patients went on to develop COVID-19 pneumonia shortly after surgery (median time to COVID-19 onset, 3.5 days), with 15 (44\%) requiring ICU admission. Ultimately, half of those (7/15) patients died in the ICU. The risks of treatment delay and impact on tumor progression should be carefully weighed given the potential for devastating outcomes should surgery be pursued, even among asymptomatic patients. Strategies to temporize tumor growth, such as induction chemotherapy, may also predispose to severe COVID-19 complications following immunosuppression. Further, treatment of HNSCC must be balanced with the current local operational environment and resources to achieve maximum patient and staff safety while minimizing morbidity. 


\section{Pulmonary Comorbidities in HNSCC}

Recently, an increased focus on competing causes of mortality among patients with HNSCC has been observed, with respiratory causes of particular interest. ${ }^{6,7,24}$ As advances in treatment modalities improve disease control and survival, the estimated number of HNSCC survivors is expected to increase. ${ }^{25}$ As a result, clinicians will be required to consider longer-term treatment effects and identify patients at highest risk for noncancer-related morbidity and mortality. Multiple studies have shown increases in adverse pulmonary outcomes among HNSCC patients compared to the general population..$^{3,6,8,26-30}$ The prevalence of respiratory comorbidities in HNSCC patients approximates 10 to $15 \%$, nearly twice as common as healthy controls. ${ }^{29,30}$ Moreover, a significant percentage $(21 \%)$ of head and neck cancer patients have moderate to severe comorbidities, second only to patients with lung $(40 \%)$ and colorectal $(25 \%)$ cancer, with a significant relationship between comorbidity severity and overall survival $(p<0.0013) .{ }^{31}$

An analysis by Rose et al. of 34,568 patients with nonmetastatic squamous cell carcinoma identified through the Surveillance Epidemiology and End Results (SEER) registry found that the most frequent causes of noncancer mortality included cardiovascular disease (28.2\%), chronic obstructive pulmonary disease (COPD, $8.5 \%)$, and cerebrovascular disease (5.6\%); lung cancer was the most common cause of second primary cancer mortality (45.8\%) (Table 1). ${ }^{7}$ On multivariable analysis, increased risk of noncancer mortality was associated with higher age, black race, unmarried status, localized disease, and nonsurgical treatment. ${ }^{7}$ Comparable results have been reported through other analyses of competing mortality, examining noncancer/comorbidity mortality and second primary cancer mortality. ${ }^{8,26,27}$ In a population-based review of 23,494 patients, Shen et al. also found cardiovascular diseases, lung cancer, COPD, and cerebrovascular disease to be the most frequent causes of competing death with rates of $28.3 \%, 10.4 \%, 8.5 \%$, and $5.7 \%$, respectively. ${ }^{8}$ These data demonstrate a disproportionate impact on the pulmonary system and increasing mortality that respiratory comorbidities may confer. Prior series have demonstrated that a large proportion of patients continue to smoke both during and after therapy. As expected, smokers have lower rates of treatment response and poorer survival compared to non-smoking counterparts, particularly if smoking continues during and after treatment. ${ }^{32-38}$

\section{Susceptibility to Adverse Respiratory Outcomes}

Patients with HNSCC are at high risk for poor respiratory outcomes due to underlying respiratory comorbidities. Kawakita et al. performed the first population-based analysis designed to compare the incidence of respiratory disease in HNSCC patients compared to the general population. In a study of 1901 head and neck cancer patients within the Utah Cancer Registry matched to 7796 noncancer patients, the authors discovered that risks of respiratory infection (HR 1.63), COPD and bronchiectasis (HR 2.65), and aspiration pneumonitis (HR 6.21) were higher among head and neck cancer survivors, even after adjusting for baseline smoking status. ${ }^{6}$ Interestingly, this increased risk persisted more than 5 years after diagnosis (Table 1 ). ${ }^{6}$ Specifically, risks of COPD and aspiration pneumonitis were more than 3-times higher among this population. Moreover, the authors demonstrated that triple modality therapy was the strongest risk factor for aspiration pneumonia. Age at diagnosis, baseline body mass index, sex, smoking status, treatment modality, primary tumor site, and stage were also identified as significant risk factors for adverse respiratory outcomes.

The risk of severe pulmonary complications remains elevated in both the immediate and long-term perioperative period. In a review of 3932 patients from a national database who underwent head and neck surgical procedures, postoperative pneumonia was the most common medical complication (3.26\%) and was associated with a mortality rate of $10.9 \%$ (OR for mortality, 4.4). ${ }^{39}$ Buitelaar et al. showed comparable outcomes in a retrospective series of 469 patients undergoing primary major head and neck ablation with cardiovascular $(12 \%)$ and respiratory (11\%) complications being the most frequent. Significant risk factors for pulmonary complications included preexisting pulmonary disease, prior myocardial infarction, and ASA grade. ${ }^{40}$ The

incidence of new respiratory comorbidities including pneumonia, asthma, and COPD has been found to be highest within the first 6 to 12 months following treatment and remains nearly two-fold higher compared to non-cancer patients. Similar findings were reported by Baxi et al. who demonstrated that mortality from 
COPD, pneumonia, and influenza continued to rise among HNSCC survivors who had lived at least 3 years after diagnosis (Table 1$)^{3}$

These findings highlight several key considerations. First, early dysphagia intervention programs may be useful in mitigating the adverse functional impacts of surgery and radiation-induced fibrosis and prevent aspiration pneumonitis. ${ }^{41}$ Second, adherence to smoking cessation is critical to reducing the risk of recurrence, second primary malignancies, and comorbid respiratory diseases ${ }^{42}$ Finally, in general, frequent disease surveillance and multidisciplinary care should remain central to the treatment and prevention of adverse pulmonary outcomes among higher risk HNSCC survivors.

\section{Pathophysiology of radiation and smoking-related injury}

\section{Radiation Effects}

RT is one of the primary treatment modalities in HNSCC. RT is believed to place HNSCC patients at increased risk of respiratory infection by two mechanisms: 1 . damage to respiratory tract cilia and 2. alteration of the microbial landscape of the upper aerodigestive tract. RT to the ciliated epithelium in the upper respiratory tract results in cellular changes that negatively affect mucociliary clearance. In a histopathological study of irradiated nasal mucosa in an animal model, Ohashi et al. observed a progressive decline in the number of cilia as epithelial metaplasia occurred several weeks after radiation. ${ }^{43}$ At the end of 8 weeks, there was complete loss of cilia. ${ }^{43}$ Similar histological findings of epithelial change and ciliary loss have been observed in irradiated larynx and nasopharynx tissue from humans ${ }^{44,45}$ Functional studies of cilia by measuring nasal secretion transport times have shown significantly slower transport in irradiated adults and children compared to healthy controls. ${ }^{46,47}$ Many patients who have been treated with radiation go on to develop chronic sinusitis. ${ }^{44,48}$

RT has also been shown to alter the microbial landscape of the oral cavity and oropharynx. Radiation to the salivary glands causes reduction in salivary flow and subsequent xerostomia. ${ }^{49,50}$ Coinciding with decreased saliva in patients treated with radiation for HNSCC, Brown et al. showed significantly altered oral flora including increases in Streptococcus mutans, Lactobacillus ,Candida, and Staphylococcus . ${ }^{49}$ Additional studies on the immunologic impact of these changes have shown alterations in the amount of immunoglobulin A (IgA), which plays a principal role in the local immune response, against virulent streptococcus species. Specifically, there was an increase in IgA activity against Streptococcus pneumoniae, a common cause of lower and upper respiratory tract infections, suggesting increased infections by this species in this population. ${ }^{50}$ Thus, it is possible that the impact of RT on oral flora may ultimately lead to downstream effects on the microbiology of respiratory infections.

\section{Smoking Effects}

The strong association between smoking and squamous cell carcinoma of the head and neck is well established. ${ }^{51}$ Smoking induces changes to the respiratory epithelium and immune system, which ultimately puts patients at increased risk of developing respiratory infections. Some of the changes that occur at the epithelial level include cell transformation, respiratory barrier disruption, mucous production, and transport alterations. ${ }^{52}$ Squamous metaplasia occurs throughout the respiratory tract with an increase in inflammatory cells and polymorphonuclear leukocytes. ${ }^{52}$ Respiratory epithelium is normally quite impermeable due to tight cell-to-cell junctions; however, these are disrupted by smoke and leads to an increase in lung permeability. ${ }^{52}$ Additionally, smoking alters respiratory secretions and transport by increasing the volume of mucous while also slowing mucociliary clearance in the upper and lower respiratory pathways. ${ }^{52}$

Smoking also alters cellular and humoral immune responses. In heavy smokers, CD4 T cell populations are decreased while CD8 cells are increased. ${ }^{53} \mathrm{CD} 4$ cells play a role in stimulating other immune cells including macrophages, B cells, and immunoglobulins; therefore, lower numbers of CD4 cells in smokers may increase their susceptibility to pulmonary infections. ${ }^{53}$ Additionally, smokers have been found to have decreased neutrophil function, increased immunosuppressive effects of macrophages, and decreased proinflammatory 
cytokines. ${ }^{53}$ In the humoral immune system, immunoglobulin populations of $\operatorname{IgA}$, IgG, and IgM have all been found to be lower than nonsmokers, thus predisposing to infection. ${ }^{53}$

\section{Viral and Bacterial Pneumonia in HNSCC}

Risks of viral and bacterial pneumonia are increased among HNSCC patients receiving chemotherapy and/or radiotherapy given the presence of unique risk factors (e.g. depressed immune function, older age, malnutrition, smoking and alcohol abuse, tumor location, and comorbidities).$^{8,26,54-56}$ Pulmonary viral infections frequently occur in patients with impaired cellular immunity, with cytomegalovirus and herpes group viruses being common sources. ${ }^{11}$ Influenza, parainfluenza, respiratory syncytial virus, rhinovirus, and adenovirus, are also recognized as frequent pulmonary pathogens, particularly among immunocompromised cancer patients with respiratory illness. ${ }^{10,12,57,58}$

Bacterial co-infection and secondary insults in HNSCC patients can lead to severe respiratory complications. Influenza-bacterial and rhinovirus-pneumococcal pneumonia have been associated with increased mortality. ${ }^{59}$ Streptococcus pneumoniae andStaphylococcus aureus are also commonly observed, accounting for as many as $35 \%$ and $28 \%$ of co-infections, respectively. ${ }^{60}$ The type of bacterial pneumonia in cancer patients depends on multiple factors including mechanism and duration of the underlying immunologic defect and whether the infection is community acquired or nosocomial.

In a review of healthcare-associated infections in HNSCC patients treated with chemotherapy and/or RT, respiratory tract infections were most common. ${ }^{55}$ Pseudomonas aeruginosarepresented the majority of gramnegative organisms (49\%) compared to gram-positive pathogens (35\%), where Staphylococcus aureuspredominated. Smoking, malnutrition, and presence of a tracheostomy tube are significant risk factors for nosocomial infections in this population. ${ }^{55,61}$ A prospective analysis by Panghal et al. revealed that HNC patients treated with chemoradiation (CRT) are prone to oral cavity bacterial infections, especially withStaphylococcus aureus.$^{62}$ Common oral flora, including anaerobes, are commonly responsible for lower respiratory infections, particularly in patients with dysphagia and aspiration. ${ }^{63,64}$

\section{Aspiration Pneumonia and Pneumonitis}

Among HNSCC patients, the etiology of aspiration, pneumonia, and pneumonitis is multifactorial and remains an important and often under-recognized source of morbidity and mortality during and following completion of treatment. In a retrospective analysis of 374 patients who received organ preservation therapy for locally advanced HNSCC, risk factors for aspiration pneumonia included poor oral hygiene, advanced $\mathrm{N}$-classification, inpatient treatment, and hypoalbuminemia. ${ }^{65}$ Treatment breaks occurred far more often in patients with aspiration pneumonia versus those without (36\% vs. $3 \%$, respectively; $p<0.01)$, which ultimately affected survival (Table 1). ${ }^{65}$ Kawai et al. reported similar findings and found that $21 \%$ (65 of 305) of patients developed pneumonia at a median of 161 days following treatment; chronic alcohol consumption, poor oral hygiene, hypoalbuminemia, coexisting malignancies (mainly esophageal and gastric cancer), and use of sleeping pills were independent predictors. ${ }^{66}$ Rates of post-treatment pneumonia range from 5 to $25 \%$, with concurrent CRT having the largest influence on its development in most studies. ${ }^{5,6,65-72}$ The sequela can be devastating with 30-day mortality rates of 20 to $30 \% .{ }^{24,73-75}$ The range in incidence of pneumonia among studies could partly be explained by patient demographics and length of follow up. In a retrospective study of 15,894 Taiwanese patients with HNSCC by Chu et al., the authors reported a lower incidence of pneumonia (5\%) within 90 days of RT initiation. ${ }^{67}$ However, only $15 \%$ of the cohort was older than age 65 , in contrast to studies with a higher proportions of elderly patients. ${ }^{65-67}$

While the risk of pneumonia in patients treated with CRT is significantly elevated during and soon after treatment, this risk continues to be elevated several years after therapy. ${ }^{6,75} \mathrm{In}$ an analysis of 3,513 patients, $\mathrm{Xu}$ et al. found that nearly one-quarter of elderly patients developed aspiration pneumonia within 5 years of CRT, with a 1 year and 5 year cumulative incidence of $15.8 \%$ and $23.8 \%$ for patients with head and neck malignancies, compared to $3.6 \%$ and $8.7 \%$ for noncancer controls, respectively. ${ }^{75}$ A $42 \%$ increased risk of death was also observed $(p<0.001)$ after controlling for confounding factors. The authors showed that 
independent risk factors for aspiration pneumonia included hypopharyngeal and nasopharyngeal tumors, increased comorbidities, older age at diagnosis, and treatment at a teaching hospital.

Notably, both of the studies from Xu et al. ${ }^{75}$ and Chu et al. ${ }^{67}$ separately identified an increased risk of aspiration pneumonia among patients receiving care at a teaching institution. This finding likely reflects a higher concentration of sicker patients with a higher degree of medical complexity at academic teaching institutions. This is important to note as many of our HNSCC population will undergo treatment at higher volume, academic centers burdened by the COVID-19 pandemic.

\section{Oral Hygiene}

Poor oral hygiene has also been separately demonstrated as an independent risk factor for the development of HNSCC and has been associated with poorer overall survival in several series. ${ }^{76-80}$ In addition to the mechanism of pathogenic oral cavity and oropharyngeal bacteria in the development of carcinoma and pneumonia, improved oral hygiene may also reduce mortality. Decrease in mortality may be associated with decreased pneumonia incidence and severity as well as the oncologic benefits conferred through routine dental and oral cavity surveillance. ${ }^{81-83}$ This further underscores the importance of maintaining a healthy oral cavity, particularly in older patients and those with previously treated HNSCC ${ }^{65,66,84}$ In all, increased attention should be paid to modifiable risk factors including smoking, oral hygiene, and nutrition status in order to mitigate aspiration risks.

\section{CONCLUSIONS :}

Primary COVID-19 infections are more likely to cause pulmonary complications (and mortality) in men, older patients, those with underlying prior or current cancers, and patients with concomitant lung conditions (such as COPD and prior smoking). Patients with head and neck cancer share similar attributes with those at highest risk for adverse outcomes with COVID-19 infection. ${ }^{3-6}$ Toxicities from CRT have been demonstrated to significantly increase rates of aspiration and pneumonia within this cohort. ${ }^{6-8}$ HNSCC patients are at increased risk for a variety of viral and bacterial pulmonary infections, which depend upon the underlying mechanism of immunosuppression. Interventions designed to decrease smoking and alcohol use, improve oral hygiene, maximize nutrition, and treat underlying medical comorbidities are central to the short and long-term management of patients with HNSCC, especially during the COVID-19 era.

\section{REFERENCES:}

1. Liang W, Guan W, Chen R, et al. Cancer patients in SARS-CoV-2 infection: a nationwide analysis in China. The Lancet Oncology.2020;21(3):335-337.

2. Zhang L, Zhu F, Xie L, et al. Clinical characteristics of COVID-19-infected cancer patients: A retrospective case study in three hospitals within Wuhan, China. Annals of oncology : official journal of the European Society for Medical Oncology. 2020.

3. Baxi SS, Pinheiro LC, Patil SM, Pfister DG, Oeffinger KC, Elkin EB. Causes of death in long-term survivors of head and neck cancer.Cancer. 2014;120(10):1507-1513.

4. Ferrier MB, Spuesens EB, Le Cessie S, Baatenburg de Jong RJ. Comorbidity as a major risk factor for mortality and complications in head and neck surgery. Archives of otolaryngology-head $\& 3$ neck surgery. $2005 ; 131(1): 27-32$.

5. Francis DO, Weymuller EA, Jr., Parvathaneni U, Merati AL, Yueh B. Dysphagia, stricture, and pneumonia in head and neck cancer patients: does treatment modality matter? The Annals of otology, rhinology, and laryngology. 2010;119(6):391-397.

6. Kawakita D, Abdelaziz S, Chen Y, et al. Adverse respiratory outcomes among head and neck cancer survivors in the Utah Cancer Survivors Study. Cancer. 2020;126(4):879-885. 
7. Rose BS, Jeong JH, Nath SK, Lu SM, Mell LK. Population-based study of competing mortality in head and neck cancer. Journal of clinical oncology : official journal of the American Society of Clinical Oncology. 2011;29(26):3503-3509.

8. Shen W, Sakamoto N, Yang L. Cancer-specific mortality and competing mortality in patients with head and neck squamous cell carcinoma: a competing risk analysis. Annals of surgical oncology.2015;22(1):264-271.

9. Sucharew H, Macaluso M. Progress Notes: Methods for Research Evidence Synthesis: The Scoping Review Approach. Journal of hospital medicine. 2019;14(7):416-418.

10. Couch RB, Englund JA, Whimbey E. Respiratory viral infections in immunocompetent and immunocompromised persons. The American journal of medicine. 1997;102(3a):2-9; discussion 25-26.

11. Rolston KV. The spectrum of pulmonary infections in cancer patients.Current opinion in oncology. 2001;13(4):218-223.

12. Stover DE, Kaner RJ. Pulmonary complications in cancer patients. CA: a cancer journal for clinicians. 1996;46(5):303-320.

13. Kamboj M, Sepkowitz KA. Nosocomial infections in patients with cancer. The Lancet Oncology. 2009;10(6):589597.

14. Ruuskanen O, Lahti E, Jennings LC, Murdoch DR. Viral pneumonia.Lancet (London, England). 2011;377(9773):12641275 .

15. Ueda M, Martins R, Hendrie PC, et al. Managing Cancer Care During the COVID-19 Pandemic: Agility and Collaboration Toward a Common Goal.Journal of the National Comprehensive Cancer Network: JNCCN.2020:1-4.

16. Livingston E, Bucher K. Coronavirus Disease 2019 (COVID-19) in Italy. Jama. 2020.

17. Onder G, Rezza G, Brusaferro S. Case-Fatality Rate and Characteristics of Patients Dying in Relation to COVID-19 in Italy.Jama. 2020.

18. WHO. Report of the WHO-China Joint Mission on Coronavirus Disease 2019 (COVID-19). https://www.who.int/docs/def source/coronaviruse/who-china-joint-mission-on-covid-19-final-report.pdf. Accessed April 12, 2020.

19. Wang H, Zhang L. Risk of COVID-19 for patients with cancer. The Lancet Oncology. 2020.

20. Chen N, Zhou M, Dong X, et al. Epidemiological and clinical characteristics of 99 cases of 2019 novel coronavirus pneumonia in Wuhan, China: a descriptive study. Lancet (London, England).2020;395(10223):507513.

21. Wang D, Hu B, Hu C, et al. Clinical Characteristics of 138 Hospitalized Patients With 2019 Novel Coronavirus-Infected Pneumonia in Wuhan, China. Jama. 2020.

22. Zhou F, Yu T, Du R, et al. Clinical course and risk factors for mortality of adult inpatients with COVID-19 in Wuhan, China: a retrospective cohort study. Lancet (London, England).2020;395(10229):1054-1062.

23. Lei S JF, Su W, et al. Clinical characteristics and outcomes of patients undergoing surgeries during the incubation period of COVID-19 infection. EClinicalMedicine. 2020. https://doi.org/10.1016/j.eclinm.2020.100331.

24. Semenov YR, Starmer HM, Gourin CG. The effect of pneumonia on short-term outcomes and cost of care after head and neck cancer surgery. The Laryngoscope. 2012;122(9):1994-2004.

25. Bray F, Ferlay J, Soerjomataram I, Siegel RL, Torre LA, Jemal A. Global cancer statistics 2018: GLOBOCAN estimates of incidence and mortality worldwide for 36 cancers in 185 countries. CA: a cancer journal for clinicians. 2018;68(6):394-424. 
26. Argiris A, Brockstein BE, Haraf DJ, et al. Competing causes of death and second primary tumors in patients with locoregionally advanced head and neck cancer treated with chemoradiotherapy. Clinical cancer research : an official journal of the American Association for Cancer Research. 2004;10(6):1956-1962.

27. Mell LK, Dignam JJ, Salama JK, et al. Predictors of competing mortality in advanced head and neck cancer. Journal of clinical oncology : official journal of the American Society of Clinical Oncology. 2010;28(1):1520.

28. Montero-Miranda PH, Ganly I. Survivorship-competing mortalities, morbidities, and second malignancies. Otolaryngologic clinics of North America. 2013;46(4):681-710.

29. Piccirillo JF, Vlahiotis A. Comorbidity in patients with cancer of the head and neck: prevalence and impact on treatment and prognosis. Current oncology reports. 2006;8(2):123-129.

30. Landis SH, El-Hariry IA, van Herk-Sukel MP, et al. Prevalence and incidence of acute and chronic comorbidity in patients with squamous cell carcinoma of the head and neck. Head $\& 3$ neck.2012;34(2):238244.

31. Piccirillo JF. Importance of comorbidity in head and neck cancer. The Laryngoscope. 2015;125(10):2242.

32. Ostroff JS, Jacobsen PB, Moadel AB, et al. Prevalence and predictors of continued tobacco use after treatment of patients with head and neck cancer. Cancer. 1995;75(2):569-576.

33. Smith J, Nastasi D, Tso R, Vangaveti V, Renison B, Chilkuri M. The effects of continued smoking in head and neck cancer patients treated with radiotherapy: A systematic review and meta-analysis.Radiotherapy and oncology : journal of the European Society for Therapeutic Radiology and Oncology. 2019;135:51-57.

34. Browman GP, Wong G, Hodson I, et al. Influence of cigarette smoking on the efficacy of radiation therapy in head and neck cancer. The New England journal of medicine. 1993;328(3):159-163.

35. van Imhoff LC, Kranenburg GG, Macco S, et al. Prognostic value of continued smoking on survival and recurrence rates in patients with head and neck cancer: A systematic review. Head \& neck. 2016;38 Suppl 1:E2214-2220.

36. Hashibe M, Brennan P, Chuang SC, et al. Interaction between tobacco and alcohol use and the risk of head and neck cancer: pooled analysis in the International Head and Neck Cancer Epidemiology Consortium. Cancer epidemiology, biomarkers 83 prevention : a publication of the American Association for Cancer Research, cosponsored by the American Society of Preventive Oncology. 2009;18(2):541-550.

37. Giraldi L, Leoncini E, Pastorino R, et al. Alcohol and cigarette consumption predict mortality in patients with head and neck cancer: a pooled analysis within the International Head and Neck Cancer Epidemiology (INHANCE) Consortium. Annals of oncology : official journal of the European Society for Medical Oncology.2017;28(11):2843-2851.

38. Spitz MR, Fueger JJ, Chamberlain RM, Goepfert H, Newell GR. Cigarette smoking patterns in patients after treatment of upper aerodigestive tract cancers. Journal of cancer education: the official journal of the American Association for Cancer Education.1990;5(2):109-113.

39. Bhattacharyya N, Fried MP. Benchmarks for mortality, morbidity, and length of stay for head and neck surgical procedures. Archives of otolaryngology-head \&3 neck surgery. 2001;127(2):127-132.

40. Buitelaar DR, Balm AJ, Antonini N, van Tinteren H, Huitink JM. Cardiovascular and respiratory complications after major head and neck surgery. Head \&3 neck. 2006;28(7):595-602.

41. Manikantan K, Khode S, Sayed SI, et al. Dysphagia in head and neck cancer. Cancer treatment reviews. 2009;35(8):724-732.

42. Mets OM, Buckens CF, Zanen P, et al. Identification of chronic obstructive pulmonary disease in lung cancer screening computed tomographic scans. Jama. 2011;306(16):1775-1781. 
43. Ohashi Y, Nakai Y, Ikeoka H, et al. Functional and morphological pathology of the nasal mucosa after x-ray irradiation. Clin Otolaryngol Allied Sci. 1988;13(6):435-446.

44. Lou PJ, Chen WP, Tai CC. Delayed irradiation effects on nasal epithelium in patients with nasopharyngeal carcinoma. An ultrastructural study. The Annals of otology, rhinology, and laryngology.1999;108(5):474480.

45. Ohyama MM, Y.; Yamamoto, S.; Fujita, T.; Taniguchi, T. Surface ultrastructure of pathological mucosa of the human larynx. Otologica Fukuoka. 1977;23:646-659.

46. Stringer SP, Stiles W, Slattery WH, 3rd, et al. Nasal mucociliary clearance after radiation therapy. The Laryngoscope. 1995;105(4 Pt 1):380-382.

47. Surico G, Muggeo P, Mappa L, et al. Impairment of nasal mucociliary clearance after radiotherapy for childhood head cancer. Head \& neck. 2001;23(6):461-466.

48. Alon EE, Lipschitz N, Bedrin L, et al. Delayed Sino-nasal Complications of Radiotherapy for Nasopharyngeal Carcinoma.Otolaryngology-head and neck surgery : official journal of American Academy of Otolaryngology-Head and Neck Surgery.2014;151(2):354-358.

49. Brown LR, Dreizen S, Handler S, Johnston DA. Effect of radiation-induced xerostomia on human oral microflora. Journal of dental research. 1975;54(4):740-750.

50. Himi T, Kukuminato Y, Kita H, Yoshioka I, Kataura A. Effect of radiotherapy on the levels of secretory immunoglobulin A against indigenous and virulent streptococci. Otolaryngology-head and neck surgery : official journal of American Academy of Otolaryngology-Head and Neck Surgery. 1997;117(5):433-437.

51. Sturgis EM, Wei Q, Spitz MR. Descriptive epidemiology and risk factors for head and neck cancer. Semin Oncol.2004;31(6):726-733.

52. Dye JA, Adler KB. Effects of cigarette smoke on epithelial cells of the respiratory tract. Thorax. 1994;49(8):825-834.

53. Arcavi L, Benowitz NL. Cigarette smoking and infection. Archives of internal medicine. 2004;164(20):22062216 .

54. Hussain M, Kish JA, Crane L, et al. The role of infection in the morbidity and mortality of patients with head and neck cancer undergoing multimodality therapy. Cancer. 1991;67(3):716-721.

55. Mirabile A, Vismara C, Crippa F, et al. Health care-associated infections in patients with head and neck cancer treated with chemotherapy and/or radiotherapy. Head $\&$ neck. 2016;38 Suppl 1:E1009-1013.

56. Merlano MC, Monteverde M, Colantonio I, et al. Impact of age on acute toxicity induced by bio- or chemo-radiotherapy in patients with head and neck cancer. Oral oncology. 2012;48(10):1051-1057.

57. Winther B. Rhinovirus infections in the upper airway.Proceedings of the American Thoracic Society. 2011;8(1):79-89.

58. Dandachi D, Rodriguez-Barradas MC. Viral pneumonia: etiologies and treatment. Journal of investigative medicine : the official publication of the American Federation for Clinical Research.2018;66(6):957-965.

59. Seki M, Kosai K, Yanagihara K, et al. Disease severity in patients with simultaneous influenza and bacterial pneumonia. Internal medicine (Tokyo, Japan). 2007;46(13):953-958.

60. Klein EY, Monteforte B, Gupta A, et al. The frequency of influenza and bacterial coinfection: a systematic review and meta-analysis.Influenza and other respiratory viruses. 2016;10(5):394-403.

61. Sepehr A, Santos BJ, Chou C, et al. Antibiotics in head and neck surgery in the setting of malnutrition, tracheotomy, and diabetes. The Laryngoscope. 2009;119(3):549-553. 
62. Panghal M, Kaushal V, Kadayan S, Yadav JP. Incidence and risk factors for infection in oral cancer patients undergoing different treatments protocols. BMC oral health. 2012;12:22.

63. Bartlett JG. Anaerobic bacterial infection of the lung. Anaerobe. 2012;18(2):235-239.

64. Langerman A, Maccracken E, Kasza K, Haraf DJ, Vokes EE, Stenson KM. Aspiration in chemoradiated patients with head and neck cancer.Archives of otolaryngology-head \& neck surgery.2007;133(12):1289-1295.

65. Shirasu H, Yokota T, Hamauchi S, et al. Risk factors for aspiration pneumonia during concurrent chemoradiotherapy or bio-radiotherapy for head and neck cancer. BMC cancer. 2020;20(1):182.

66. Kawai S, Yokota T, Onozawa Y, et al. Risk factors for aspiration pneumonia after definitive chemoradiotherapy or bio-radiotherapy for locally advanced head and neck cancer: a monocentric case control study. BMC cancer. 2017;17(1):59.

67. Chu CN, Muo CH, Chen SW, Lyu SY, Morisky DE. Incidence of pneumonia and risk factors among patients with head and neck cancer undergoing radiotherapy. BMC cancer. 2013;13:370.

68. Hunter $\mathrm{KU}$, Lee $\mathrm{OE}$, Lyden $\mathrm{TH}$, et al. Aspiration pneumonia after chemo-intensity-modulated radiation therapy of oropharyngeal carcinoma and its clinical and dysphagia-related predictors. Head $\mathcal{G}$ neck.2014;36(1):120-125.

69. Mortensen HR, Jensen K, Grau C. Aspiration pneumonia in patients treated with radiotherapy for head and neck cancer. Acta oncologica (Stockholm, Sweden). 2013;52(2):270-276.

70. Nguyen NP, Frank C, Moltz CC, et al. Aspiration rate following chemoradiation for head and neck cancer: an underreported occurrence.Radiotherapy and oncology : journal of the European Society for Therapeutic Radiology and Oncology. 2006;80(3):302-306.

71. Chen SW, Yang SN, Liang JA, Lin FJ. The outcome and prognostic factors in patients with aspiration pneumonia during concurrent chemoradiotherapy for head and neck cancer. European journal of cancer care. 2010;19(5):631-635.

72. Eisbruch A, Lyden T, Bradford CR, et al. Objective assessment of swallowing dysfunction and aspiration after radiation concurrent with chemotherapy for head-and-neck cancer. Int J Radiat Oncol Biol Phys. 2002;53(1):23-28.

73. Komiya K, Ishii H, Umeki K, et al. Impact of aspiration pneumonia in patients with communityacquired pneumonia and healthcare-associated pneumonia: a multicenter retrospective cohort study. Respirology (Carlton, Vic). 2013;18(3):514-521.

74. Lanspa MJ, Jones BE, Brown SM, Dean NC. Mortality, morbidity, and disease severity of patients with aspiration pneumonia. Journal of hospital medicine. 2013;8(2):83-90.

75. Xu B, Boero IJ, Hwang L, et al. Aspiration pneumonia after concurrent chemoradiotherapy for head and neck cancer. Cancer.2015;121(8):1303-1311.

76. Chang CC, Lee WT, Hsiao JR, et al. Oral hygiene and the overall survival of head and neck cancer patients. Cancer medicine.2019;8(4):1854-1864.

77. Farquhar DR, Divaris K, Mazul AL, Weissler MC, Zevallos JP, Olshan AF. Poor oral health affects survival in head and neck cancer. Oral oncology. 2017;73:111-117.

78. Friemel J, Foraita R, Gunther K, et al. Pretreatment oral hygiene habits and survival of head and neck squamous cell carcinoma (HNSCC) patients. BMC oral health. 2016;16:33.

79. Hashim D, Sartori S, Brennan P, et al. The role of oral hygiene in head and neck cancer: results from International Head and Neck Cancer Epidemiology (INHANCE) consortium. Annals of oncology : official journal of the European Society for Medical Oncology.2016;27(8):1619-1625. 
80. Zeng XT, Leng WD, Zhang C, Liu J, Cao SY, Huang W. Meta-analysis on the association between toothbrushing and head and neck cancer. Oral oncology. 2015;51(5):446-451.

81. Sjogren P, Nilsson E, Forsell M, Johansson O, Hoogstraate J. A systematic review of the preventive effect of oral hygiene on pneumonia and respiratory tract infection in elderly people in hospitals and nursing homes: effect estimates and methodological quality of randomized controlled trials. Journal of the American Geriatrics Society.2008;56(11):2124-2130.

82. Sjogren P, Wardh I, Zimmerman M, Almstahl A, Wikstrom M. Oral Care and Mortality in Older Adults with Pneumonia in Hospitals or Nursing Homes: Systematic Review and Meta-Analysis. Journal of the American Geriatrics Society. 2016;64(10):2109-2115.

83. Muller F. Oral hygiene reduces the mortality from aspiration pneumonia in frail elders. Journal of dental research. 2015;94(3 Suppl):14s-16s.

84. Yokota T, Tachibana H, Konishi T, et al. Multicenter phase II study of an oral care program for patients with head and neck cancer receiving chemoradiotherapy. Supportive care in cancer : official journal of the Multinational Association of Supportive Care in Cancer.2016;24(7):3029-3036.

\section{Hosted file}

HED-20-0536_Table_1 (3).docx available at https://authorea.com/users/312328/articles/443249respiratory-and-pulmonary-complications-in-head-and-neck-cancer-patients-an-evidence-basedreview-for-the-covid-19-era 